\title{
Versuche zur Synthese des 3, 4-8, 9-Dibenz-pyrens
}

\author{
Ton \\ Richard Weitzenböck ${ }^{1}$ \\ Aus dem Chemischen Institut der $k$. k. Universität Graz \\ Herausgegeben von Christian Seer2
}

(Vorgelegt in der Sitzung am 10. Jänner 1918)

Die nach Überwindung großer Schwierigkeiten erfolgreich durchgeführte Synthese des Pyrens ${ }^{3}$ legte den Gedanken nahe, den hier gangbar befundenen Weg auch zur Darstellung von Derivaten dieses Körpers, besonders von höher anellierten Kohlenwasserstoffen, die den Pyrenkern enthalten, nutzbar zu machen.

Die ersten in dieser Richtung unternommenen Versuche, die durch den Ausbruch des Krieges unterbrochen wurden, zielten auf die Darstellung des 3, 4-8, 9-Dibenz-pyrens hin. An Stelle des $o$-Ditolyls bei der Pyrensynthese wurde hier das in der vorhergehenden Mitteilung von Scholl beschriebene 1, 1'-Dimethyl-2, $2^{\prime}$-binaphtyl benutzt, welches die beiden dem Pyrenkern anzugliedernden Sechsringe bereits vorgebildet. enthält.

1 Fürs Vaterland gefallen am 19. Dezember 1915 in Galizien.

2 Auf Veranlassung Prof. Scholl's, als dessen Assistent Weitzenböck seinerzeit die Arbeiten über das Pyren begonnen hat, habe ich es übernommen, die leider nicht zum Abschlup geiangten Versuche meines langjährigen Kollegen und Freundes auf Grund seiner nachgelassenen Aufzeicl. nungen zu veröffentlichen.

Seer.

3 M. 34, 193 (1913). 
Das 1, 1'-Dimethyl-2, 2'-binaphtyl wurde in der Seitenkette bromiert, das Brom durch Cyan ersetzt und durch Verseifen des 1, 1'-Dicyanomethyl-2, 2'-binaphtyls die $2,2^{\prime}$-Binaphtylen-1, $1^{\prime}$-diessigsäure (I) erhalten, welche auf folgendem Wege in das gesuchte 3, 4-8, 9-Dibenz-pyren (IV) übergeführt werden sollte:

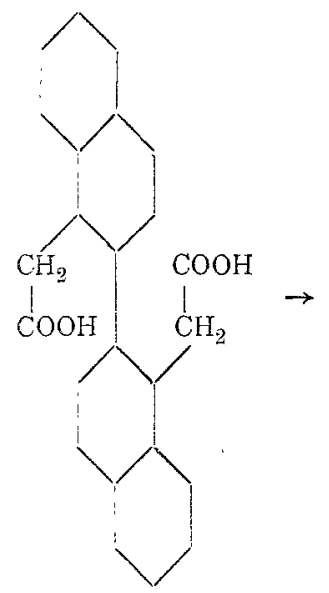

I.

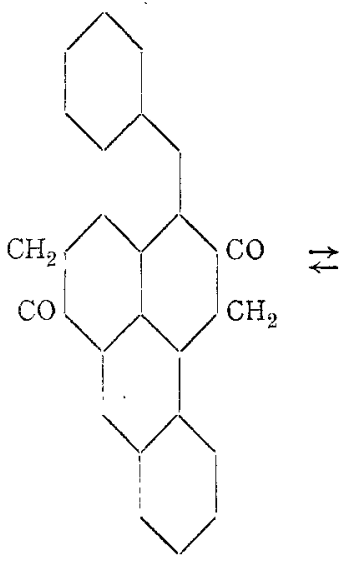

II.

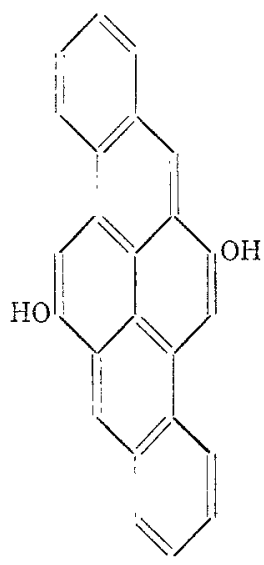

III. ${ }^{1}$

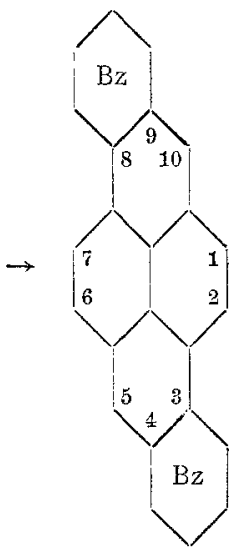

IV.

1 Fußnote nebenstehend. 


\section{Versuche.}

\section{Kohlenwasserstoff $\mathrm{C}_{22} \mathrm{H}_{16}$ (?) als Nebenprodukt der Darstellung des 1, 1'-Dimethyl-2, 2'-binaphtyls.}

Wenn man die Benzolmutterlaugen von der Darstellung des in der vorhergehenden Mitteilung von Scholl beschriebenen 1, 1'-Dimethyl-2, 2'-binaphtyls aus rohem 1-Methyl-2-jodnaphtalin vom Benzol befreit und den schwarzen, zähen Sirup aus einer Retorte destilliert, dann geht zunächst eine geringe Menge einer farblosen Flüssigkeit (1-Methylnaphtalin), dann ein krystallinisch erstarrendes Destillat über, das noch nicht untersucht wurde. Beim weiteren Erhitzen bis zum völligen Verkohien des Retorteninhaltes und Erweichen des Glases destilliert ein rotbraunes, schon im Retortenhals erstarrendes Öl. Durch abwechselndes mehrmaliges Umkrystallisieren dieser Fraktion aus Xylol und Eisessig wurden farblose Blättchen erhalten, deren Schmelzpunkt allmählich von 295 bis $297^{\circ}$ auf 314 bis $316^{\circ}$ anstieg. Nach Sublimation dieser Substanz im Kohlendioxydstrom und nochmaliger Krystallisation aus Eisessig lag der Schmelzpunkt bei 309 bis $312^{\circ}$. Der Körper liefert in benzolischer Lösung, mit überschüssiger Pikrinsäure zusammengebracht, ein in schönen ziegelroten Nadeln krystallisierendes Pikrat vom Schmelzpunkt 199 bis $201^{\circ}$, aus dem durch Zersetzung mit verdünntem Ammoniak farblose Nadeln vom Schmelzpunkt 308 bis $310^{\circ}$ erhalten wurden. Neuerdings ins Pikrat übergeführt, schmolz dieses bei $199^{\circ}$ bis $200^{\circ}$. Es scheint also sowohl der Schmelzpunkt des

1 In der Vitteilung über Pyren (1. c.) ist bei der Valenzformel (XXY1) des 1, 6-Dioxypyrens ein Druckfehler übersehen worden. Die Formel ist richtig in folgender Weise zu schreiben:

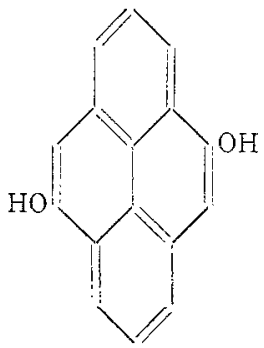


Pikrats als auch der des Kohlenwasserstoffes bei weiterem Umkrystallisieren konstant $z \mathrm{u}$ bleiben.

Die von Hansgirg nach der Pregl'schen Methode ausgeführte Mikroanalyse einer noch nicht völlig aschefreien Substanzprobe (Schmelzpunkt 302 bis $311^{\circ}$ ) ergab folgende Werte:

3. $.84 \mathrm{mg}$ Substanz $\left(0.026 \mathrm{mg}\right.$ Asche) gaben $13.08 \mathrm{mg}^{\mathrm{CO}} \mathrm{CO}_{2}, 1.78 \mathrm{mg} \mathrm{H}_{2} \mathrm{O}$.

Ber. für $\mathrm{C}_{22} \mathrm{H}_{16}=280 \cdot 1 \mathrm{C} 94 \cdot 26, \mathrm{H} 5 \cdot 76 \%$.

Ber. fuir $\mathrm{C}_{22} \mathrm{H}_{14}=278.1 \mathrm{C} 94.93$, $\mathrm{H} 5.07010$.

Gef. C $94 \cdot 27$, H $5 \cdot 26 \%$.

Aus der Analyse läßt sich keine Entscheidung zwischen den beiden Formeln $\mathrm{C}_{22} \mathrm{H}_{16}$ und $\mathrm{C}_{22} \mathrm{H}_{14}$ treffen. Die ursprüngliche Vermutung, daß der Kohlenwasserstoff mit Picen $\left(\mathrm{C}_{22} \mathrm{H}_{14}\right.$, Schmelzpunkt $\left.350^{\circ}\right)$ identisch sei, scheint sich nicht zu bestätigen, da der Schmelzpunkt um etwa $40^{\circ}$ tiefer liegt. ${ }^{1}$ ) Es gewinnt deshalb die Formel $\mathrm{C}_{2.2} \mathrm{H}_{16}$ an Wahrscheinlichkeit. Ein Kohlenwasserstoff dieser Zusammensetzung könnte -

1) Die Arbeit von H. Meyer und A. Hofmann »Über Pyrokondensationen in der aromatischen Reibe «, [M. 37, 682 (1916)] wurde mir erst während der Drucklegung dieser Versuche im Original zugänglich. Nach den dort (S. 715) beschriebenen großen Schwierigkeiten bei der Identifizierung von unreinem Picen, scheint es doch nicht ausgeschlossen, daß der Kohlenwasserstoff von Weitzen böck - wie er selber früher angenommen hatte ebenfalls Picen war, dessen Schmelzpunkt durch geringe Mengen hartnäckig anhaftender Verunreinigungen herabgedrückt wurde. Jedenfalls dürfte sich diese Frage durch die Anwendung der H. Meyer'schen Kondensationsmethode auf das 1.1'-Dimethyl-2.2'-binaphthyl entscheiden lassen.

Auch das in der folgenden Abliandlung beschriebene 3.4-5.6-Dibenzphenanthren wirce sich wohl nach dieser Methode aus 2.2'-Dimethyl1.1'-binaphthyl in etwas größerer Menge erhalten lassen.
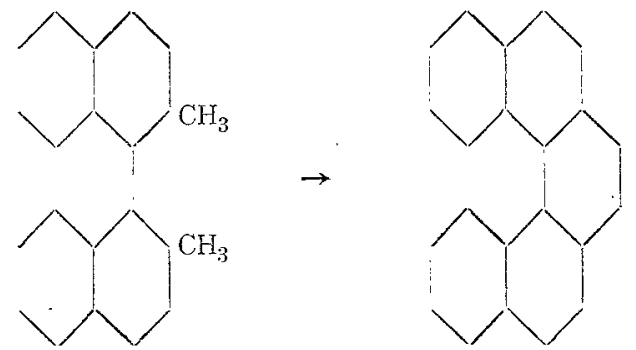

Die Bildungsweise wäre analog der nach $H$. Meyer sehr glatt verlaufenden Daistellung von Phenanthren aus o-Ditolyl. 
wenn man von einer Umlagerung der Atomgruppen innerhalb des Moleküls absieht - aus 2, 2'-Dimethyl-1, 1'-binaphtyl auf pyrogenem Wege in folgender Weise entstanden sein:

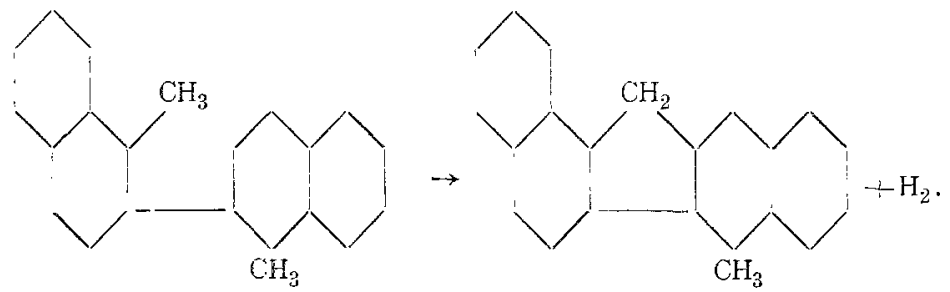

\section{1, 1'-Dibromomethyl-2, 2'-binaphtyl.}

In eine siedende Lösung von $10 \mathrm{~g} 1,1^{\prime}$-Dimethyl-2, $2^{\prime}$-binaphtyl in $20 \mathrm{~g}$ Nitrobenzol werden $4 \mathrm{~cm}^{3}$ Brom (=4 At.) mittels eines langsamen Kohlensäurestromes dampfförmig eingeleitet. Die dunkle Lösung verdickt sich dabei und scheidet beim Erkalten harte Krystallkrusten aus. Diese werden abgesaugt und zweimal unter Zusatz von Tierkohle aus Benzol umkrystallisiert. Man erhält das 1, 1'-Dibromomethyl-2, 2'-binaphtyl in farblosen, zu Drusen verwachsenen, dicken Nadeln, die sich ziemlich leicht in Benzol lösen und konstant bei 200 bis $201^{\circ}$ schmelzen.

Analyse (Substanz bei $110^{\circ}$ getrocknet):

4.755 $\mathrm{mls}_{\mathrm{s}}$ Substanz gaben $4.09 \mathrm{mzr} \mathrm{AgBr}$.

Ber. für $\mathrm{C}_{22} \mathrm{H}_{16} \mathrm{Br}_{2}=440 \mathrm{Br} 36 \cdot 340,0$. Gef. $\operatorname{Br} 36 \cdot 600^{\prime}$.

\section{1, 1'-Dicyanomethyl-2, 2'-binaphtyl.}

In eine Lösung von $2 g$ Cyankalium in wenig Wasser werden nach dem Verdünnen mit etwa 3 Volumteilen Alkohol $5 g$ gepulvertes 1, 1'-Dibromomethyl-2, 2'-binaphtyl bei Siedetemperatur eingetragen. Nach kurzem Sieden läßt man auf $60^{\circ}$ abkühlen und erhält bei dieser Temperatur noch 4 Stunden. Nach dieser Zeit hat sich die Hauptmenge des gebildeten Nitrils in schönen, farblosen Krystallen (Schmelzpunkt 253 bis $254^{\circ}$ ) ausgeschieden, die ohne weiteres abfiltriert werden. Aus den Mutterlaugen gewinnt man noch eine kleine Menge 
durch Verdünnen mit Wasser. Ausbeute quantitativ. Sollte in der erhaltenen Substanz noch Brom nachzuweisen sein, so kann man die Reaktion durch nochmaliges Erwärmen mit alkoholischer Kaliumcyanidlösung zu Ende führen.

$1,1^{\prime}$-Dicyanomethyl-2, $2^{\prime}$-binaphtyl ist schwer löslich in Eisessig und sehr schwer (nur bei längerem Kochen) in Benzol. Die Analysensubstanz wurde dreimal aus Benzol umkrystallisiert. Sie schmilzt dann konstant bei $264^{\circ}$.

Analyse (Substanz bei $120^{\circ}$ getrocknet):

$0.1016 \mathrm{~g}$ Substanz gaben $0.3229 \mathrm{~g} \mathrm{CO}_{2}, 0.0461 \mathrm{~g} \mathrm{H}_{2} \mathrm{O}$.

Ber. fiir $\mathrm{C}_{24} \mathrm{H}_{16} \mathrm{~N}_{2}=332 \cdot 2$ C $86 \cdot 70$, $\mathrm{H} 4 \cdot 86^{\circ} 0_{0}$.

Gef. C $86 \cdot 68$, H $5 \cdot 190: 0$.

\section{2, 2'-Binaphtylen-1, 1'-diessigsäure (Formel I).}

$\lg 1,1^{\prime}$-Dicyanomethyl-2,2'-binaphtyl wurde mit $3 g$ festem Ätzkali und $20 \mathrm{~cm}^{3}$ Alkohol im Bombenrohr 4 Stunden auf $150^{\circ}$ erhitzt. Nach dem Erkalten wurde der Inhalt des Rohres, eine geringe Menge eines festen Körpers in gelblicher Flüssigkeit, suspendiert, mit Wasser herausgespült und der Alkohol durch Erwärmen auf dem Dampfbad entfernt. Hierauf wurde vom Ungelösten abfiltriert und das Filtrat durch Kochen mit Tierkohle entfärbt. Aus der filtrierten erkalteten Lösung fällt die gebildete 2, 2'-Binaphtyen-1, $1^{\prime}$-diessigsäure auf Zusatz von Salzsäure in weißen Flocken aus.

Duich zweimaliges Umkrystallisieren aus Toluol, in dem die Substanz schwer löslich ist, wurden farblose mikroskopische Nädelchen vom Schmelzpunkt 285 bis $287^{\circ}$ erhalten. Eine Analyse wurde nicht ausgeführt.

\section{Anhang.}

Vorversuche zu einer neuen Pyrensynthese. o-Jodstyrylchlorid $\mathrm{C}_{6} \mathrm{H}_{4} \backslash \mathrm{CH}: \mathrm{CHCl}^{1}$

$5 \stackrel{g}{0}$-Jodzimmtsäure wurden in $1 / 2 l$ Schwefelkohlenstoff aufgeschlämmt und so lange Chlor eingeleitet, bis die Säure in

I Nach dem Verfahren von H. Biliz für w-Chlorstyrol, Lieb. Ann, 296. $266(1897)$. 
Lösung gegangen ist, was längere Zeit in Anspruch nimmt. Aus der Lösung scheiden sich Krystallnadeln der o Jodphenyldichlorpropionsäure aus. Man läßt sie über Nacht stehen, dampft den Schwefelkohlenstoff aus einem mäßig warmen Bade $a b$, versetzt den schön krystallinen Rückstand mit Wasser und Soda im Überschuß und behandelt mit Wasserdampf. Das o-Jodstyrylchlorid destilliert als farbloses Ö1. Es wird mit Äther aufgenommen und nach Entfernung des Äthers unter vermindertem Druck destilliert. Es siedet konstant bei $151^{\circ}$ bei $24 \mathrm{~mm}$, nur die ersten Tropfen sind rötlichbraun. Unter gewöhnlichem Druck siedet es bei etwa $270^{\circ}$ unter starker Jodabscheidung und Zersetzung. Analyse:

$0.1581 \mathrm{~g}$ Substanz (Rohprodukt vor der Vakulumdestillation) gaben 0.2237 . $\mathrm{AgCl}+\mathrm{Ag} \mathrm{J}$.

$0.2057 \mathrm{~g}$ Substanz (rein) gaben $0.2745 \mathrm{~g} \mathrm{CO}_{2} .0 .0545 \mathrm{~g} \mathrm{H}_{2} \mathrm{O}$.

Ber. fuir $\mathrm{C}_{8} \mathrm{H}_{6} \mathrm{ClJ}(264 \cdot 5) \mathrm{Ag} \mathrm{Cl}+\mathrm{AgJ} 143 \cdot 03, \mathrm{C} \mathrm{36} \cdot 30, \mathrm{H} \mathrm{2.290'}$. Gef. $\mathrm{AgCl}+\mathrm{AgJ} 141 \cdot \overline{\mathrm{s}}, \mathrm{C} 36 \cdot 40$, H $2.980^{\prime}$. 